\begin{tabular}{|c|l|}
\hline Title & A symptotic behavior of type I blowup solutions to a parabolic-elliptic system of drift-diffusion type \\
\hline Author(s) & Yoshikazu, Giga; Noriko, Mizoguchi; Takasi, Senba \\
\hline Citation & Hokkaido University Preprint Series in Mathematics, 968, 1-30 \\
\hline Issue Date & 2010-9-3 \\
\hline DOI & 10.14943/84115 \\
\hline Doc URL & http://hdl.handle.net/2115/69775 \\
\hline Type & bulletin (article) \\
\hline File Information & pre968.pdf \\
\hline
\end{tabular}

Instructions for use 


\title{
Asymptotic behavior of type I blowup solutions to a parabolic-elliptic system of drift-diffusion type
}

\author{
Yoshikazu Giga, Noriko Mizoguchi and Takasi Senba
}

Abstract A Cauchy problem for a parabolic-elliptic system of drift-diffusion type is considered. The problem is formally of the form

$$
U_{t}=\nabla \cdot\left(\nabla U-U \nabla(-\Delta)^{-1} U\right) .
$$

This system describes a mass-conserving aggregation phenomenon including gravitational collapse and bacterial chemotaxis. Our concern is the asymptotic behavior of blowup solutions when the blowup is type I in the sense that its blowup rate is the same as the corresponding ordinary differential equation $y_{t}=y^{2}$ (up to a multiple constant). It is shown that all type I blowup is asymptotically (backward) self-similar provided that the solution is radial, nonnegative when the blowup set is a singleton and the space dimension is greater than or equal to three.

2000 Mathematics Subject Classification. 35K55, 35K57, 92C17. 


\section{Introduction}

In this paper, we consider the blowup of radial solutions to the system

$$
\begin{cases}U_{t}=\nabla \cdot(\nabla U-U \nabla V) & \text { in } \mathbf{R}^{N} \times(0, T), \\ 0=\Delta V+U & \text { in } \mathbf{R}^{N} \times(0, T), \\ U(x, 0)=U_{0}(x) \geq 0 & \text { in } \mathbf{R}^{N}\end{cases}
$$

where $N \geq 3$ and $U_{0} \in L^{\infty}\left(\mathbf{R}^{N}\right)$ is radially symmetric. We first recall the definition of a solution $(U, V)$ to (1.1). For any radial function $U_{0} \in L^{\infty}\left(\mathbf{R}^{N}\right)$, there exists a unique radial function $U \in C\left([0, T) ; L^{\infty}\left(\mathbf{R}^{N}\right)\right)$ satisfying

$$
\begin{aligned}
& U(x, t)=\int_{\mathbf{R}^{N}} \mathcal{G}(x-\tilde{x}, t) U_{0}(\tilde{x}) d \tilde{x} \\
& \quad+\int_{0}^{t} \int_{\mathbf{R}^{N}}\left[\nabla_{\tilde{x}} \mathcal{G}(x-\tilde{x}, t-\tilde{t}) \cdot \frac{\tilde{x}}{\omega_{N}|\tilde{x}|^{N}} \int_{|\hat{x}| \leq|\tilde{x}|} U(\hat{x}, \tilde{t}) d \hat{x}\right] U(\tilde{x}, \tilde{t}) d \tilde{x} d \tilde{t}
\end{aligned}
$$

in $\mathbf{R}^{N} \times[0, T)$ for some constant $T>0$, where $\omega_{N}$ is the area of the unit sphere in $\mathbf{R}^{N}$ and $\mathcal{G}$ is the Gauss kernel of $\partial_{t}-\Delta$ in $\mathbf{R}^{N}$. Define $V$ by

$$
V(x, t)=C-\int_{0}^{|x|} \frac{1}{\omega_{N} r^{N-1}} \int_{|\tilde{x}|<r} U(\tilde{x}, t) d \tilde{x} d r
$$

where $C$ is an arbitrary constant. Then $(U, V)$ satisfies (1.1) in the classical sense by the parabolic regularity argument. We call $(U, V)$ defined above a solution of (1.1). It is immediate that $U>0$ in $\mathbf{R}^{N} \times(0, T)$ if $U_{0} \geq 0$ and $U_{0} \not \equiv 0$ in $\mathbf{R}^{N}$.

The system (1.1) was introduced in [7] as a simplified system of

$$
\left\{\begin{array}{l}
U_{t}=\nabla \cdot(\nabla U-U \nabla V), \\
V_{t}=\Delta V-V+U
\end{array}\right.
$$

The system (1.3) is a model for several biological problems (e.g.[12]) and physical problems (e.g. [2]).

In a biological problem, the system (1.3) describes that cellular slime molds aggregate owing to the motion of the cells which move towards higher concentration of a chemical substance produced by themselves. In the model, $U(x, t)$ and $V(x, t)$ represent the density of cells and the concentration of the chemical substance, respectively.

We say that a solution $(U, V)$ blows up at $t=T$ if $\limsup _{t \rightarrow T}|U(t)|_{\infty}=\infty$ with the $L^{\infty}$-norm in $\mathbf{R}^{N}$. Moreover if for $p \in \mathbf{R}^{N}$ there exists a sequence $\left\{\left(x_{n}, t_{n}\right)\right\} \subset$ 
$\mathbf{R}^{N} \times(0, T)$ with $x_{n} \rightarrow p$ and $t_{n} \rightarrow T$ as $n \rightarrow \infty$ such that $U\left(x_{n}, t_{n}\right) \rightarrow \infty$ as $n \rightarrow \infty$, then $p$ is called a blowup point of $(U, V)$. The set of all blowup points is called the blowup set of $(U, V)$ and denoted by $B(U, V)$. It was shown in $[9,16,17]$ that the systems (1.1), (1.3) have blowup solutions. We say that a solution $(U, V)$ of (1.1) defined in $\mathbf{R}^{N} \times(-\infty, 0)$ is backward self-similar if $\lambda^{2} U\left(\lambda x, \lambda^{2} t\right)=U(x, t)$ in $\mathbf{R}^{N} \times(-\infty, 0)$ for each $\lambda>0$. The system (1.1) has radial backward self-similar blowup solutions (see [8] for $N=3$ and [16] for $N \geq 3$ ).

Let $(U, V)$ be a radial solution to (1.1). Put

$$
u(\xi, t)=\frac{1}{\omega_{N} \xi^{N}} \int_{|x|<\xi} U(x, t) d x \quad \text { for }(\xi, t) \in[0, \infty) \times[0, T) .
$$

Then $u$ satisfies

$$
\frac{\partial u}{\partial t}=\frac{\partial^{2} u}{\partial \xi^{2}}+\frac{(N+1)}{\xi} \frac{\partial u}{\partial \xi}+u\left(\xi \frac{\partial u}{\partial \xi}+N u\right) \quad \text { in }(0, \infty) \times(0, T)
$$

and

$$
\frac{\partial u}{\partial \xi}(0, t)=0 \quad \text { for } t \in(0, T) .
$$

It is immediate that $u$ is positive in $[0, \infty) \times(0, T)$. The definition of blowup point and blowup set for $u$ is similarly done to above. For $T>0$, put

$$
w(r, s)=(T-t) u(\xi, t)
$$

with

$$
r=(T-t)^{-1 / 2} \xi \quad \text { and } \quad s=-\log (T-t) .
$$

Then $w$ satisfies

$$
\begin{cases}\frac{\partial w}{\partial s}=\frac{\partial^{2} w}{\partial r^{2}}+\left(\frac{N+1}{r}-\frac{r}{2}\right) \frac{\partial w}{\partial r}-w+w\left\{r \frac{\partial w}{\partial r}+N w\right\} & \text { in }(0, \infty) \times\left(s_{0}, \infty\right), \\ w\left(r, s_{0}\right)=w_{0}(r) \equiv T u\left(T^{1 / 2} r, 0\right) & \text { in }[0, \infty),\end{cases}
$$

where $s_{0}=-\log T$. For $\alpha>0$, let $\varphi_{\alpha}$ be a solution to

$$
\varphi^{\prime \prime}+\left(\frac{N+1}{r}-\frac{r}{2}\right) \varphi^{\prime}-\varphi+\varphi\left(r \varphi^{\prime}+N \varphi\right)=0 \quad \text { in }(0, r(\alpha))
$$

with $\varphi^{\prime}(0)=0$ and $\varphi(0)=\alpha$, where $r(\alpha)=\sup \left\{r>0: 0<\varphi_{\alpha}(\tilde{r})<\infty\right.$ for $\left.\tilde{r} \in[0, r)\right\}$. Let $\mathcal{S}=\{\alpha>0: r(\alpha)=\infty\}$. A solution $u$ of $(1.5)$ defined in $[0, \infty) \times(-\infty, 0)$ is 
backward self-similar if $\lambda^{2} u\left(\lambda \xi, \lambda^{2} t\right)=u(\xi, t)$ in $[0, \infty) \times(-\infty, 0)$ for each $\lambda>0$. It is equivalent to $u(\xi, t)=(-t)^{-2} \varphi_{\alpha}\left((-t)^{-1 / 2} \xi\right)$ for $(\xi, t) \in[0, \infty) \times(-\infty, 0)$ with some $\alpha \in \overline{\mathcal{S}}$. It is immediate that $\varphi_{\infty}$ defined by

$$
\varphi_{\infty}(r)=\frac{2}{r^{2}} \quad \text { for } r>0
$$

is a singular solution of (1.7).

A solution $(U, V)$ of (1.1) is said to exhibit type I blowup at $t=T$ if there exists a constant $K>0$ such that $|U(t)|_{\infty} \leq K(T-t)^{-1}$ for $t \in[0, T)$. In other words, $|w(s)|_{\infty}$ is uniformly bounded in $\left[s_{0}, \infty\right)$ for the corresponding solution $w$ of (1.6). In [6], an asymptotic behavior of $w(s)$ associated with type I blowup solution as $s \rightarrow \infty$ was studied for (1.1) in $B_{1}$ with the boundary conditions

$$
\frac{\partial U}{\partial \nu}-U \frac{\partial V}{\partial \nu}=0 \quad \text { and } \quad V=0 \quad \text { on } \partial B_{1} \times(0, T),
$$

where $B_{R}=\left\{x \in \mathbf{R}^{N}:|x|<R\right\}$ for $R>0$ and $\nu$ is the outward normal unit vector. They showed that if $u(\xi, 0)$ satisfies

$$
u_{\xi}(\xi, 0) \leq 0 \quad \text { for } \xi \in[0,1)
$$

and

$$
u_{\xi \xi}(\xi, 0)+\frac{N+1}{\xi} u_{\xi}(\xi, 0)+u(\xi, 0)\left\{\xi u_{\xi}(\xi, 0)+N u(\xi, 0)\right\} \geq 0 \quad \text { for } \xi \in(0,1)
$$

then $w(s)$ converges to $\varphi_{\alpha}$ locally uniformly in $[0, \infty)$ as $s \rightarrow \infty$ for some $\hat{\alpha} \in \mathcal{S}$ with $z\left(\varphi_{\hat{\alpha}}-\varphi_{\infty}\right)=1$. Here for a function $f \not \equiv 0$ on $[a, b]$ with $0 \leq a<b \leq \infty$, let $z(f:[a, b))$ be the supremum over all $j$ such that there exist $a \leq r_{1}<r_{2}<\cdots<r_{j+1}<b$ with $f\left(r_{i}\right) \cdot f\left(r_{i+1}\right)<0$ for $i=1,2, \cdots, j$. Denote by $z(f)$ for simplicity when $b=\infty$. It was given in [15] that if $3 \leq N \leq 9$, then there exists a sequence $\left\{\alpha_{n}\right\} \subset \mathcal{S}$ with $\alpha_{n} \rightarrow \infty$ as $n \rightarrow \infty$ such that $z\left(\varphi_{\alpha_{n}}-\varphi_{\infty}\right) \rightarrow \infty$ as $n \rightarrow \infty$. The condition (1.9) excludes the possibility of the convergence of $w(s)$ to $\varphi_{\alpha}$ with $\alpha \neq \hat{\alpha}$ as $s \rightarrow \infty$.

Put $\overline{\mathcal{S}}=\mathcal{S} \cup\{0\}$. We first obtain the convergence of $w(s)$ corresponding to type I blowup solution to $\varphi_{\alpha}$ for some $\alpha \in \overline{\mathcal{S}}$ under no extra assumptions.

Theorem 1.1 Suppose that a radial solution $(U, V)$ of (1.1) undergoes type $I$ blowup at $t=T$ and that $B(U, V)=\{0\}$. Let $w$ be the corresponding solution of (1.6). Then, $w(s)$ converges to $\varphi_{\alpha}$ locally uniformly as $s \rightarrow \infty$ for some $\alpha \in \overline{\mathcal{S}}$. Under an additional assumption (1.8), the above conclusion holds with $\alpha \in \mathcal{S}$. 
This theorem means that a solution $u$ of (1.5) corresponding to a radial solution of (1.1) undergoing type I blowup at $t=T$ is asymptotically backward self-similar near $\xi=0$ as $t \rightarrow T$.

Throughout the present paper, we denote $L_{l o c}^{\infty}([0, \infty))$ and $C_{l o c}^{i}([0, \infty))$ by $L_{l o c}^{\infty}$ and $C_{l o c}^{i}$, respectively. Let $\omega\left(w_{0}\right)$ be the omega-limit set of the solution $w$ of (1.6) in Theorem 1.1, that is,

$$
\begin{aligned}
& \omega\left(w_{0}\right)=\left\{W: w\left(s_{n}\right) \rightarrow W \text { in } L_{l o c}^{\infty} \text { as } n \rightarrow \infty\right. \\
& \left.\quad \text { for a sequence }\left\{s_{n}\right\} \text { with } s_{n} \rightarrow \infty \text { as } n \rightarrow \infty\right\} .
\end{aligned}
$$

The equation (1.6) without the convection term rwwr appears in the study of blowup problem for a semilinear heat equation. In that case, the standard argument of infinite dimensional dynamical system based on a Lyapunov function plays an essential role to show that the omega-limit set consists of stationary solutions. On the other hand, the convection term $r w w_{r}$ prevents us from an easy construction of a Lyapunov function. In [6], a well-known method to treat a parabolic equation in one-dimensional bounded interval introduced by [19] was modified to get a Lyapunov function in implicit form with an advantage of bounded interval. However the method needed so complicated calculations. We make use of the analyticity with respect to the spatial variable to avoid such hard calculations, namely, we require no Lyapunov function.

Let us sketch our proof. It is immediate that $\omega\left(w_{0}\right)$ is nonempty by the parabolic regularity argument. We first show that $W(0)$ is constant for $W \in \omega\left(w_{0}\right)$ by use of the intersection comparison between $w$ and $\varphi_{\alpha}$ for $\alpha \in \mathcal{S}$ (Lemma 4.3). Based on the fact, we show

$$
\frac{\partial^{k} w}{\partial s^{k}}(0, s) \rightarrow 0 \quad \text { as } s \rightarrow \infty
$$

for each positive integer $k$ (Lemma 4.4). This leads us to

$$
\frac{\partial^{i+j+1}}{\partial r^{j} \partial s^{i+1}} w(0, s) \rightarrow 0 \quad \text { as } s \rightarrow \infty
$$

for any nonnegative integers $i, j$ (Lemma 4.5). There exist $S>s_{0}$ and $R>0$ such that

$$
\sup \left\{\frac{1}{k !}\left|\frac{\partial^{k}}{\partial r^{k}} w_{s}(r, s)\right| \cdot r^{k}: r \in(0, R) \text { and } s \geq S_{0}\right\} \rightarrow 0 \quad \text { as } k \rightarrow \infty
$$

(Corollary 2.1). This is derived from a general theorem on spatial analyticity (Theorem 2.1). Let

$$
\begin{aligned}
\mathcal{V}=\left\{V: w_{s}\left(s_{n}\right)\right. & \rightarrow V \text { in } L_{l o c}^{\infty} \text { as } n \rightarrow \infty \\
& \text { for a sequence } \left.\left\{s_{n}\right\} \text { with } s_{n} \rightarrow \infty \text { as } n \rightarrow \infty\right\} .
\end{aligned}
$$


Take $V \in \mathcal{V}$ arbitrarily. By the parabolic regularity argument, for each nonnegative integer $j$ there exists a sequence $\left\{s_{n}\right\}$ with $s_{n} \rightarrow \infty$ such that $w_{s}\left(s_{n}\right) \rightarrow V$ in $C_{l o c}^{j}$ as $n \rightarrow \infty$. It follows from (1.10) that

$$
\frac{d^{j}}{d r^{j}} V(0)=0 \quad \text { for } j=1,2,3, \cdots
$$

By the Taylor expansion, we get

$$
\begin{aligned}
w_{s}(r, s)= & w_{s}(0, s)+\frac{\partial}{\partial r} w_{s}(0, s) \cdot r+\frac{1}{2} \frac{\partial^{2}}{\partial r^{2}} w_{s}(0, s) \cdot r^{2} \\
& +\cdots+\frac{1}{(k-1) !} \frac{\partial^{k-1}}{\partial r^{k-1}} w_{s}(0, s) \cdot r^{k-1}+\frac{1}{k !} \frac{\partial^{k}}{\partial r^{k}} w_{s}(r \theta, s) \cdot r^{k}
\end{aligned}
$$

with some $\theta \in(0,1)$ for $r>0$ and $s>s_{0}$. This implies

$$
\begin{aligned}
V(r)= & V(0)+\frac{d}{d r} V(0) \cdot r+\frac{1}{2} \frac{d^{2}}{d r^{2}} V(0) \cdot r^{2} \\
& +\cdots+\frac{1}{(k-1) !} \frac{d^{k-1}}{d r^{k-1}} V(0) \cdot r^{k-1}+\frac{1}{k !} \frac{d^{k}}{d r^{k}} V(r \theta) \cdot r^{k}
\end{aligned}
$$

for $r>0$. We now obtain that $V$ is analytic at $r=0$ by (1.11) and hence $V \equiv 0$ from (1.12). Since for each $W \in \omega\left(w_{0}\right)$, there exists a sequence $\left\{s_{n}\right\}$ with $s_{n} \rightarrow \infty$ as $n \rightarrow \infty$ such that $w\left(s_{n}\right) \rightarrow W$ and $w_{s}\left(s_{n}\right) \rightarrow 0$ in $C_{l o c}^{2}$ as $n \rightarrow \infty$, we get

$$
W_{r r}+\frac{N+1}{r} W_{r}-\frac{r}{2} W_{r}-W+W\left(r W_{r}+N W\right)=0 \quad \text { in }(0, \infty) .
$$

Since $W(0)$ is constant for $W \in \omega\left(w_{0}\right)$, we obtain $\omega\left(w_{0}\right)=\left\{\varphi_{\alpha}\right\}$ for some $\alpha \in \overline{\mathcal{S}}$. This implies that $w(s) \rightarrow \varphi_{\alpha}$ in $L_{l o c}^{\infty}$ as $s \rightarrow \infty$.

For a solution $u$ of $(1.5)$, let $U(\xi, t)=\xi^{1-N}\left(\xi^{N} u\right)_{\xi}$ and $V_{\xi}(\xi, t)=-\xi u(\xi, t)$ for $(\xi, t) \in[0, \infty) \times[0, T)$. Then $(U, V)$ satisfies $(1.1)$. We say that a solution $(U, V)$ of (1.1) defined in $[0, \infty) \times(-\infty, 0)$ is backward self-similar if $\lambda^{2} U\left(\lambda \xi, \lambda^{2} t\right)=U(\xi, t)$ in $[0, \infty) \times(-\infty, 0)$ for each $\lambda>0$. Put $U_{\alpha}(\xi, t)=(-t)^{-1} \Phi_{\alpha}\left((-t)^{-1 / 2} \xi\right)$ and $\left(V_{\alpha}\right)_{\xi}(\xi, t)=$ $-(-t)^{-1} \xi \varphi_{\alpha}\left((-t)^{-1 / 2} \xi\right)$ for $(\xi, t) \in[0, \infty) \times(-\infty, 0)$ for $\alpha \in \overline{\mathcal{S}}$, where $\Phi_{\alpha}(r)=$ $r\left(\varphi_{\alpha}\right)_{r}(r)+N \varphi_{\alpha}(r)$ for $r \geq 0$. It is immediate that $\left(U_{\alpha}, V_{\alpha}\right)$ is a backward self-similar solution of (1.1). It follows from (1.1) and (1.2) that

$$
\begin{aligned}
U(\xi, t) & =-\left(V_{\xi \xi}+\frac{N-1}{\xi} V_{\xi}(\xi, t)\right) \\
& =\xi u_{\xi}(\xi, t)+N u(\xi, t) .
\end{aligned}
$$

Therefore we easily get the following result from Theorem 1.1. 
Corollary 1.1 Suppose that a radial solution $(U, V)$ of (1.1) undergoes type $I$ blowup at $t=T$ and that $B(U, V)=\{0\}$. Let $\varphi_{\alpha}$ be as in Theorem 1.1. Then we have

$$
(T-t) U\left((T-t)^{1 / 2} r, t\right) \rightarrow r\left(\varphi_{\alpha}\right)_{r}(r)+N \varphi_{\alpha}(r) \quad \text { in } L_{l o c}^{\infty} \text { as } t \rightarrow T .
$$

In other words, $(U, V)$ is asymptotically backward self-similar near $\xi=0$ as $t \rightarrow T$.

This paper is organized as follows: In Section 2, we get an estimate on the analyticity with respect to space variable. In section 3 , we study the behavior of $\varphi_{\alpha}(r)$ as $r \rightarrow \infty$ for $\alpha \in \mathcal{S}$. Section 4 is devoted to the proof of the main theorem.

\section{Estimates related to analyticity}

We shall state spatial analyticity of solutions of parabolic equations with analytic nonlinearity. Such a statement has been proved by [4] for a general system of fully nonlinear parabolic equations. Although it is stated in his paper as Theorem 2 of [4], we give here a version for semilinear equations to avoid complification. We also give a different proof based on Hölder estimates since such estimates simplifies induction argument significantly.

We first recall parabolic Hölder norms. For a domain $D$ in $\mathbf{R}^{N} \times \mathbf{R}$ and a function $u$ on $D$ we denote a parabolic $\mu$ - Hölder seminorm on $D$ by

$$
[u]_{\mu, D}=\sup \left\{|u(x, t)-u(y, s)| /\left(|x-y|^{\mu}+|t-s|^{\mu / 2}\right):(x, t),(y, s) \in D\right\},
$$

where $0<\mu<1$. For our convenience we denote the maximum norm by

$$
[u]_{0, D}=\sup \{|u(x, t)|:(x, t) \in D\}
$$

The parabolic $\mu$ - Hölder norm is now defined by

$$
|u|_{\mu, D}=[u]_{\mu, D}+[u]_{0, D}
$$

We use a standard convention of multi-indices $\alpha=\left(\alpha_{1}, \ldots, \alpha_{N}\right)$ for order of spatial partial derivatives, i.e.,

$$
\partial_{x}^{\alpha}=\partial_{x_{1}}^{\alpha_{1}} \cdots \partial_{x_{N}}^{\alpha_{N}}, \partial_{x_{j}}=\partial / \partial x_{j}, \quad j=1,2, \ldots, N
$$

and $|\alpha|=\alpha_{1}+\cdots+\alpha_{N}$. To simplify the notation we also use the convention that

$$
\left[w^{(k)}\right]_{\mu, D}=\sup _{|\alpha|=k}\left[\partial_{x}^{\alpha} w\right]_{\mu, D}
$$


for higher derivatives. Of course, $\left[w^{(h)}\right]_{0, D}$ and $\left|w^{(h)}\right|_{\mu, D}$ are defined similarly. Note that these quantities only contain spatial derivatives.

We recall a parabolic ball of radius $r$ centered at $(0,0)$ defined by

$$
Q(R)=B_{R} \times\left(-R^{2}, 0\right), \quad B_{R}=\left\{x \in \mathbf{R}^{n}:|x|<R\right\} .
$$

We now state spatial analyticity of solutions for semilinear parabolic equations with analytic nonlinearity.

Theorem 2.1 Let $w$ be a smooth solution of

$$
\frac{\partial w}{\partial t}=\Delta w+f(x, w, \nabla w) \quad \text { in } Q(1) .
$$

assume that there exist $M>0$ and $\tilde{M}>0$ such that

$$
[w]_{0, Q(1)} \leq M, \quad\left[w^{(1)}\right]_{0, Q(1)} \leq \tilde{M}
$$

Assume that $f$ is analytic in a neighborhood of

$$
W=\left\{(x, p, q) \in \mathbf{R}^{2 N+1}: x \in B_{1},|p| \leq M,|q| \leq \tilde{M}\right\}
$$

Then there exist constants $C, d>0$ depending only on $M, \tilde{M}, N, f$ such that

$$
\left[w^{(k)}\right]_{0, Q(1 / 2)} \leq C k^{k} d^{k}
$$

i.e.,

$$
\left|\partial_{x}^{\alpha} w(x, t)\right| \leq C k^{k} d^{k} \quad \text { in } \quad Q(1 / 2)
$$

By the Stirling formula we have

$$
k !=\sqrt{2 \pi} k^{k+\frac{1}{2}} e^{-k+\theta_{k} / 12 k}
$$

with some constant $\theta_{k} \in(0, k)$. Theorem 2.1 with this representation of $k$ ! yields the following strong convergence result for $w_{s}$ of (1.6) as $s \rightarrow \infty$. This is a key for the proof of Theorem1.1.

Corollary 2.1 Let $w$ be a uniformly bounded global solution of (1.6). Then there exist $S>s_{0}$ and $R>0$ such that

$$
\lim _{k \rightarrow \infty} \sup \left\{\frac{1}{k !}\left|\frac{\partial^{k}}{\partial r^{k}} w_{s}(r, s)\right| \cdot r^{k}: r \in(0, R), s \geq S\right\}=0
$$


Proof of Theorem 2.1. We may assume that $M^{\prime}=\left[w^{(2)}\right]_{0, Q(1)}<\infty$ by replacing $Q(1)$ by a smaller parabolic ball $Q(r)$.

Our goal is to prove that for a fixed Hölder exponent $\mu \in(0,1)$

$$
\left[w^{(k)}\right]_{\mu, \delta} \leq A(H k / \delta)^{k+\mu}, k=1,2, \ldots
$$

with some positive constants $A$ and $H$ independent of $k$ and $\delta \in(0,1 / 2)$ (depending only on $\mu, M, \tilde{M}$ and $N$ ), where

$$
[u]_{\mu, \delta}=[u]_{\mu, Q(1-\delta)}
$$

We recall a few a priori estimates for the heat equation. Let $u$ be a solution of

$$
\partial_{t} u-\Delta u=h \text { in } Q\left(1-\delta^{\prime}\right), \quad 0<\delta^{\prime}<\delta
$$

which is smooth in a parabolic neighborhood of $\overline{Q\left(1-\delta^{\prime}\right)}$. The solution $u$ is of the form

$$
u=G[h]+P[u] .
$$

Here $G$ is the Green operator with the Dirichlet condition and $P$ is the Poisson operator with the boundary data $u$. In other words $G[h]$ is the unique solution of

$$
v_{t}-\Delta v=h \quad \text { in } \quad Q\left(1-\delta^{\prime}\right)
$$

with $v=0$ on a parabolic boundary $\partial_{p} Q\left(1-\delta^{\prime}\right)$, i.e.,

$$
\partial_{p} Q\left(1-\delta^{\prime}\right)=\partial B\left(1-\delta^{\prime}\right) \times\left(-\left(1-\delta^{\prime}\right)^{2}, 0\right) \cup \overline{B\left(1-\delta^{\prime}\right)} \times\left\{-\left(1-\delta^{\prime}\right)^{2}\right\}
$$

and $P[\varphi]$ is the unique solution of

$$
Z_{t}-\Delta Z=0 \text { in } Q\left(1-\delta^{\prime}\right), \quad v=\varphi \text { on } \partial_{p} Q\left(1-\delta^{\prime}\right)
$$

For $G$ a classical Schauder estimate implies

$$
\left|(G[h])^{(2)}\right|_{\mu, \delta^{\prime}} \leq c_{1}|h|_{\mu, \delta^{\prime}}
$$

with a constant $c_{1}$ depending only on $N$ and $\mu$, and independent of $h$ and $\delta^{\prime}$ provided that $0 \leq \delta^{\prime}<1 / 2$. For the proof see e.g. [13]. The constant $c_{2}$ depends on domains but in our case $Q\left(1-\delta^{\prime}\right)$ is homothesic in parabolic scale for all $\delta^{\prime}<1$ so if the size is pinched from above and below, the constant $c_{1}$ can be taken uniform. (Note that $G$ 
and $P$ depends on $\delta^{\prime}$ but we suppress its dependence.)

For $P$ a direct computation of fundamental solution ([5], [11], [13]) implies that

$$
\left[P[\varphi]^{(j)}\right]_{0, \delta} \leq \frac{m_{j}}{\left(\delta-\delta^{\prime}\right)^{j}}[\varphi]_{0, \delta^{\prime}}, \quad j=0,1,2, \ldots
$$

with $m_{j}$ independent of $\varphi, \delta, \delta^{\prime}$ provided that $0<\delta^{\prime}<\delta<1 / 2$ and depending only on $j$ and $N$. (The dependence on $j$ for large $j$ is $j^{j}$ but we do not use such an estimate.) Here $[\varphi]_{0, \delta^{\prime}}$ denotes the maximum norm of $\varphi$ over $\partial_{p} Q\left(1-\delta^{\prime}\right)$, where $\varphi$ is defined only on the boundary $\partial_{p} Q\left(1-\delta^{\prime}\right)$. By a trivial interpolation inequality

$$
[v]_{\mu, \delta} \leq 2^{1-\mu}\left[v^{(1)}\right]_{0, \delta}^{\mu}[v]_{0, \delta}^{1-\mu}
$$

the derivative estimate for $P$ yields the Hölder estimate

$$
\left[P[\varphi]^{(j)}\right]_{\mu, \delta} \leq \frac{c_{2}}{\left(\delta-\delta^{\prime}\right)^{j+\mu}}[\varphi]_{0, \delta^{\prime}}
$$

with a constant $c_{2}$ depending only on $N$ and $\mu$.

We are now ready to carry out the proof of (2.1). By our assumptions and (2.3) we know that $(2.1)$ holds for $k=1$. We argue by induction. Suppose that (2.1) is valid for $k=r-1$ for $r \geq 2$. We take $\delta^{\prime}$ such that $\delta-\delta^{\prime}=\delta / r$ and use the representation to formula for $\partial_{x}^{\alpha} w$ :

$$
\partial_{x}^{\alpha} w=G\left[\partial_{x}^{\alpha} F\right]+P\left[\partial_{x}^{\alpha} w\right]
$$

with $F(x)=f(x, w(x), \nabla w(x))$ and $|\alpha|=r-2$. By (2.2) and (2.4) we have

$$
\left[w^{(r)}\right]_{\mu, \delta} \leq c_{1}\left|F^{(r-2)}\right|_{\mu, \delta^{\prime}}+c_{2}\left(\frac{r}{\delta}\right)^{2+\mu}\left[w^{(r-2)}\right]_{0, \delta^{\prime}}
$$

We shall use an interpolation inequality (proved later in Lemma 2.1)

$$
\left[u^{(1)}\right]_{0, \delta^{\prime}} \leq c_{3}\left[u^{(1)}\right]_{\mu, \delta^{\prime}}^{1-\mu}[u]_{\mu, \delta^{\prime}}^{\mu}
$$

with $c_{3}$ depending only on $N$ and $\mu$ to derive estimates for maximum norm. Our induction assumption implies that

$$
\left[w^{(k)}\right]_{0, \delta^{\prime}} \leq c_{3} A\left(H k / \delta^{\prime}\right)^{k} \text { for } 0 \leq k \leq r-1 \text {. }
$$

For the first term a calculation similar to the proof of Lemma 1 in [4] together with (2.7) implies

$$
\left[\partial_{x}^{\alpha} F\right]_{\mu, \delta^{\prime}} \leq C A\left(H(r-1) / \delta^{\prime}\right)^{r-1+\mu},\left[\partial_{x}^{\alpha} F\right]_{0, \delta^{\prime}} \leq C A\left(H(r-1) / \delta^{\prime}\right)^{r-1}
$$


with $C$ independent of $\delta^{\prime}$ and $r$ provided that $H$ is taken large but largeness is independent of $\delta^{\prime}$ and $r$. The constant $C$ depends on analyticity of $f$. Since we estimate Hölder norm, we frequently invoke a trivial inequality

$$
[g h]_{\mu, \delta^{\prime}} \leq[g]_{0, \delta^{\prime}}[h]_{\mu, \delta^{\prime}}+[g]_{\mu, \delta^{\prime}}[h]_{0, \delta^{\prime}}
$$

to derive (2.8). Note that the highest derivative term of $w$ is in $\partial_{x}^{\alpha} F$ is $\partial f / \partial(\nabla w)$. $\partial_{x}^{\alpha} \nabla w$, so $\partial_{x}^{\alpha} F$ only includes derivatives of $w$ up to $r-1$ order. Since we may assume that $H>1$, we conclude from (2.8) that

$$
\begin{gathered}
\left|F^{(r-2)}\right|_{\mu, \delta^{\prime}} \leq 2 C A\left(H(r-1) / \delta^{\prime}\right)^{r-1+\mu} \\
=2 C A(H r / \delta)^{r-1+\mu} .
\end{gathered}
$$

Combining (2.5), (2.7) and (2.9), we have

$$
\left[w^{(r)}\right]_{\mu, \delta^{\prime}} \leq c_{1} 2 C A\left(H r / \delta^{\prime}\right)^{r-1+\mu}+c_{2} c_{3} A\left(H(r-2) / \delta^{\prime}\right)^{r-2}(r / \delta)^{2+\mu} .
$$

Taking $H$ larger (independent of $r$ and $\delta \in(0,1 / 2)$ ), say,

$$
2 c_{1} C H^{-1} \cdot \frac{1}{2}+c_{2} c_{3} H^{-2} \leq H,
$$

we observe that

$$
\left[w^{(r)}\right]_{\mu, \delta} \leq A(H r / \delta)^{r-1+\mu}
$$

We have proved (2.1).

Our conclusion follows from (2.1) with interpolation inequality (2.6).

We now give a short proof for an interpolation inequality (2.6) for parabolic Hölder norm.

Lemma 2.1 Let $r_{0}$ and $R_{0}$ be positive constants such that $r_{0}<R_{0}$. For $\mu \in(0,1)$ and $N=1,2, \ldots$ there is a constant $c=c\left(r_{0}, R_{0}, \mu, N\right)$ such that

$$
\left[w^{(1)}\right]_{0} \leq c\left[w^{(1)}\right]_{\mu}^{1-\mu}[w]_{\mu}^{\mu}
$$

for all $w \in C(\overline{Q(R)})$ such that $\nabla w \in C(\overline{Q(R)})$ and $r_{0}<R<R_{0}$, where $Q(R) \subset$ $\mathbf{R}^{N} \times \mathbf{R}$. Here $[h]_{\mu}$ denotes the norm on $\overline{Q(R)}$, i.e. $[h]_{\mu, Q(R)}$.

Proof. We first prove that

$$
\left[\partial_{x_{i}} w\right]_{0, U} \leq C\left[\partial_{x_{i}} w\right]_{\mu, U}^{1-\mu}[w]_{\mu, U}^{\mu} \text { for } w \in C^{\mu}(U), \quad U=\mathbf{R}^{N} \times(-\infty, 0) .
$$


It suffices to prove that

$$
\left[\partial_{x_{i}} w\right]_{0, U} \leq c^{\prime}\left(\lambda^{\mu}\left[\partial_{x_{i}} w\right]_{\mu, U}+\lambda^{\mu-1}[w]_{\mu, U}\right)
$$

for all $\lambda>0$ with some constant $c^{\prime}$ independent of $\lambda$ and $w$. By scaling it suffices to prove the case $\lambda=1$. For this purpose it suffices to prove

$$
\left[\partial_{x_{i}} w\right]_{0, Q} \leq C_{1}^{\prime}\left(\left[\partial_{x_{i}} w\right]_{\mu, Q}+[w]_{\mu, Q}\right)
$$

in a cube $Q \subset U$.

Suppose that this inequality would not hold. Then there would exist a sequence of function $w_{j}$ such that

$$
\left[\partial_{x_{i}} w_{j}\right]_{0}=1 \text { and }\left[\partial_{x_{i}} w_{j}\right]_{\mu, Q}+\left[w_{j}\right]_{\mu, Q} \leq 1 / j .
$$

By Ascoli-Arzelà theorem $\partial_{x_{i}} w_{j}$ converges uniformly in $Q$ to some function $g$. Since $\left[\partial_{x_{i}} w_{j}\right]_{\mu, Q} \rightarrow 0, g$ is a function independent of $x_{i}$. However, since $\left[w_{j}\right]_{\mu, Q} \rightarrow 0$, we have $w_{j} \rightarrow$ const. $(j \rightarrow \infty)$. This is absurd.

We thus proved that

$$
\left[w^{(1)}\right]_{0, U} \leq c\left[w^{(1)}\right]_{\mu, U}^{1-\mu}[w]_{\mu, U}^{\mu} .
$$

To prove the same inequality on $Q(R)\left(r_{0}<R<R_{0}\right)$ instead of $U$ it suffices to extend function on $Q(R)$ to a function on $U$. For a given function $f \in C^{1}(\overline{Q(R)})$ we define its extension to $\mathbf{R}^{N} \times\left(-R^{2}, 0\right)$ by a kind of reflection

$$
\tilde{f}(x, t)=2 f(R x /|x|, t)-f\left(R^{2} x /|x|^{2}, t\right) \quad \text { for } \quad|x| \geq R
$$

We further extend for $t \leq-R^{2}$ by a similar method, i.e.

$$
\bar{f}(x, t)=2 \tilde{f}\left(x,-R^{2}\right)-\tilde{f}\left(x,-R^{4} / t\right) \text { for } \quad t \leq-R^{2} .
$$

It is clear that

$$
[\bar{f}]_{\mu, U} \leq a[f]_{\mu, Q(R)},\left[\bar{f}^{(1)}\right]_{\mu, U} \leq b[f]_{\mu, Q(R)}
$$

with a constant $a$ and $b$ depending only on $r_{0}, R_{0}, \mu$ and $N$. Thus (2.10) yields the desired estimate for $Q(R)$ so the proof of Lemma2.1 is now complete.

\section{Properties of positive solutions of (1.7)}

For $\alpha>0$, let $\varphi_{\alpha}$ be a solution to

$$
\left\{\begin{array}{l}
\varphi^{\prime \prime}+\left(\frac{N+1}{r}-\frac{r}{2}\right) \varphi^{\prime}-\varphi+\varphi\left(r \varphi^{\prime}+N \varphi\right)=0 \quad \text { in }(0, r(\alpha)) \\
\varphi^{\prime}(0)=0, \quad \varphi(0)=\alpha,
\end{array}\right.
$$


where $r(\alpha)=\sup \left\{r>0: 0<\varphi_{\alpha}(\tilde{r})<\infty\right.$ for $\left.\tilde{r} \in[0, r)\right\}$. Let $\mathcal{S}=\{\alpha>0: r(\alpha)=$ $\infty\}$.

The following is the main result in this section.

Proposition 3.1 Let $N \geq 3$. For $\alpha \in \mathcal{S}$, the limit $\Lambda_{\alpha} \equiv \lim _{r \rightarrow \infty} r^{2} \varphi_{\alpha}(r)$ exits. Furthermore, if $\alpha_{1}, \alpha_{2} \in \mathcal{S}$ and $\alpha_{1} \neq \alpha_{2}$, then $\Lambda_{\alpha_{1}} \neq \Lambda_{\alpha_{2}}$.

Put $\kappa=1 / N$ and let

$$
L_{\kappa}(\phi)=\phi^{\prime \prime}+\frac{N+1}{r} \phi^{\prime}-r\left(\frac{1}{2}-\frac{1}{N}\right) \phi^{\prime}+\phi .
$$

Let $L_{\rho}^{2}$ be the Lebesgue measurable functions $f$ on $[0, \infty)$ satisfying

$$
\int_{0}^{\infty} f(r)^{2} r^{N+1} \rho(r) d r<\infty
$$

where

$$
\rho(r)=\exp \left(-\frac{N-2}{4 N} r^{2}\right) \quad \text { for } r \geq 0 .
$$

For a nonnegative integer $j$, let $\lambda_{j}^{\kappa}$ be the $j$ th eigenvalue of $L_{\kappa}(\phi)=-\lambda \phi$, and let $\phi_{j}^{\kappa}$ be the $j$ th eigenfunction normalized in $L_{\rho}^{2}$ such that $\phi_{j}^{\kappa}(r)>0$ for $r \gg 1$. Here and henceforth, for positive constants $a$ and $b a \gg b$ denotes that $a / b$ is sufficiently large. It is known that

$$
\lambda_{j}^{\kappa}=\frac{N-2}{N} j-1 \text { and } \phi_{j}^{\kappa}(r)=(-1)^{j} c_{j} S_{j}^{N / 2}\left(\frac{N-2}{4 N} r^{2}\right),
$$

respectively. Here

$$
c_{j}=\left\{\int_{0}^{\infty}\left(S_{j}^{N / 2}\left(\frac{N-2}{4 N} r^{2}\right)\right)^{2} r^{N+1} \rho(r) d r\right\}^{-1 / 2}
$$

and $S_{j}^{a}$ is the Sonine's polynomial of order $a$ for $j=0,1,2, \cdots$. Then we see

$$
\begin{aligned}
\phi_{j}^{\kappa}(r) & =c_{j}\left(\frac{N-2}{4 N} r^{2}\right)^{j}\left(1+O\left(r^{-2}\right)\right) \quad \text { as } r \rightarrow \infty \\
\text { and } & \\
\frac{d}{d y} \phi_{j}^{\kappa}(r) & =\frac{2 j}{r} c_{j}\left(\frac{N-2}{4 N} r^{2}\right)^{j}\left(1+O\left(r^{-2}\right)\right) \quad \text { as } r \rightarrow \infty .
\end{aligned}
$$


Lemma 3.1 Let $\alpha \in \mathcal{S} \backslash\{\kappa\}$. If $z\left(\varphi_{\alpha}-\kappa\right)=\infty$, there exists a sequence $\left\{r_{n}\right\} \subset$ $[0, \infty)$ with $r_{n} \rightarrow \infty$ as $n \rightarrow \infty$ such that $\varphi_{\alpha}\left(r_{n}\right)=\kappa$ and that $\varphi_{\alpha}<\kappa$ in $\left(r_{2 n}, r_{2 n+1}\right)$ for $n=1,2,3, \cdots$.

Proof. If $z\left(\varphi_{\alpha}-\kappa\right)=\infty$, then there exists $\left\{r_{n}\right\} \subset[0, \infty)$ such that $\varphi_{\alpha}\left(r_{n}\right)=\kappa$ and that $\varphi_{\alpha}<\kappa$ in $\left(r_{2 n}, r_{2 n+1}\right)$ for $n=1,2,3, \cdots$. If $\left\{r_{n}\right\}$ is bounded, then there exists $R>0$ with $r_{n} \rightarrow R$ as $n \rightarrow \infty$ taking a subsequence if necessary. If $\varphi_{\alpha}$ is bounded in $[0,2 R]$, we get $\varphi_{\alpha}(R)=\kappa$ and $\varphi_{\alpha}^{\prime}(R)=0$ by the elliptic regularity argument. This implies $\alpha=\kappa$ by the uniqueness of solution to the initial value problem for the ordinary differential equation. This contradicts that $\alpha \in \mathcal{S} \backslash\{\kappa\}$, which completes the proof.

Lemma 3.2 If $\alpha \in \mathcal{S} \backslash\{\kappa\}$, then $z\left(\varphi_{\alpha}-\kappa\right)<\infty$.

Proof. On the contrary, we assume $z\left(\varphi_{\alpha}-\kappa\right)=\infty$. Put $\Phi=\kappa-\varphi_{\alpha}$. The function $\Phi$ satisfies

$$
\Phi^{\prime \prime}+\frac{N+1}{r} \Phi^{\prime}-\left(\frac{1}{2}-\frac{1}{N}\right) r \Phi^{\prime}-\Phi+N\left(\kappa+\varphi_{\alpha}\right) \Phi-r \Phi \Phi^{\prime}=0
$$

and

$$
\frac{1}{g} \frac{d}{d r}\left(g \frac{d \Phi}{d r}\right)-\Phi+N\left(\kappa+\varphi_{\alpha}\right) \Phi-r \Phi \Phi^{\prime}=0
$$

where

$$
g(r)=r^{N+1} \exp \left(-\frac{N-2}{4 N} r^{2}\right) .
$$

For $j=0,1,2, \cdots$, we see

$$
\frac{1}{g} \frac{d}{d r}\left(g \frac{d \phi_{j}^{\kappa}}{d r}\right)+\phi_{j}^{\kappa}=-\lambda_{j}^{\kappa} \phi_{j}^{\kappa} .
$$

Let $\left\{r_{n}\right\}$ be the sequence in Lemma 3.1. It follows from (3.19) that

$$
\int_{r_{2 n}}^{r_{2 n+1}} \frac{d}{d r}\left(g \frac{d \phi_{j}^{\kappa}}{d r}\right) \Phi d r+\int_{r_{2 n}}^{r_{2 n+1}} \phi_{j}^{\kappa} \Phi g d r+\lambda_{j}^{\kappa} \int_{r_{2 n}}^{r_{2 n+1}} \phi_{j}^{\kappa} \Phi g d r=0 .
$$

Therefore we have

$$
-\int_{r_{2 n}}^{r_{2 n+1}}\left(\lambda_{j}^{\kappa}+1\right) \Phi g \phi_{j}^{\kappa} d r=\int_{r_{2 n}}^{r_{2 n+1}} \frac{d}{d r}\left(g \frac{d \phi_{j}^{\kappa}}{d r}\right) \Phi d r>\int_{r_{2 n}}^{r_{2 n+1}} \phi_{j}^{\kappa}\left(\Phi^{\prime} g\right)^{\prime} d r .
$$


Applying (3.17) to this inequality yields

$$
\int_{r_{2 n}}^{r_{2 n+1}} r \phi_{j}^{\kappa} g \Phi \Phi^{\prime} d r<-\lambda_{j}^{\kappa} \int_{r_{2 n}}^{r_{2 n+1}} \phi_{j}^{\kappa} g \Phi d r<0 \quad \text { for } n \gg 1 .
$$

It follows from (3.15) and (3.16) that

$$
\left(r \phi_{j}^{\kappa} g\right)^{\prime}=\left\{N+2+2 j c_{j}\left(1+O(1) \frac{1}{r^{2}}\right)-\left(\frac{1}{2}-\frac{1}{N}\right) r^{2}\right\} \phi_{j}^{\kappa} g \quad \text { for } r \gg 1 .
$$

Therefore we obtain

$$
\begin{aligned}
& \int_{r_{2 n}}^{r_{2 n+1}} r \phi_{j}^{\kappa} g \Phi \Phi^{\prime} d r=-\frac{1}{2} \int_{r_{2 n}}^{r_{2 n+1}}\left(r \phi_{j}^{\kappa} g\right)^{\prime} \Phi^{2} d r \\
& =-\frac{1}{2} \int_{r_{2 n}}^{r_{2 n+1}}\left\{N+2+2 j c_{j}\left(1+O(1) \frac{1}{r^{2}}\right)-\left(\frac{1}{2}-\frac{1}{N}\right) r^{2}\right\} \phi_{j}^{\kappa} g \Phi^{2} d r>0
\end{aligned}
$$

for $n \gg 1$. This contradicts (3.20), which completes the proof.

Lemma 3.3 If $\alpha \in \mathcal{S}$, then $\sup _{r \geq 0} \varphi_{\alpha}(r)<\infty$.

Proof. On the contrary, we assume $\lim \sup _{r \rightarrow \infty} \varphi_{\alpha}(r)=\infty$ for some $\alpha \in S$. By Lemma 3.2, we have $\varphi_{\alpha}(r)>\kappa$ for $r \gg 1$. If $\varphi_{\alpha}$ has a local minimum at $R \gg 1$, then

$$
\varphi_{\alpha}^{\prime \prime}(R)=\varphi_{\alpha}(R)-N\left(\varphi_{\alpha}(R)\right)^{2}<0 .
$$

This contradicts that $\varphi_{\alpha}(R)$ is a local minimum, which implies $\lim _{r \rightarrow \infty} \varphi_{\alpha}(r)=\infty$ and $\varphi_{\alpha}^{\prime}(r) \geq 0$ for $r \gg 1$. Hence we get

$$
\varphi_{a}^{\prime \prime}(r)+2 r \varphi_{a}^{\prime}(r)<0 \quad \text { for } r \geq R
$$

with some $R \gg 1$. This inequality leads us to

$$
\varphi_{\alpha}^{\prime}(r)<\varphi_{\alpha}^{\prime}(R) \frac{R^{2}}{r^{2}} \quad \text { for } r>R .
$$

This contradicts that $\lim _{\sup _{r \rightarrow \infty}} \varphi_{\alpha}(r)=\infty$, which completes the proof.

Lemma 3.4 If $\alpha>0$, then $\varphi_{\alpha}(r)<\infty$ for $r \in(0, r(\alpha)]$. 
Proof. On the contrary, we assume $\lim \sup _{r \rightarrow r(\alpha)} \varphi_{\alpha}(r)=\infty$. By Lemma 3.1, we see $r(\alpha)<\infty$. If $\varphi_{\alpha}$ has a local minimum at $R \in(r(\alpha) / 2, r(\alpha))$, it follows from (3.13) that

$$
0 \leq \varphi_{\alpha}^{\prime \prime}(R)=\varphi_{\alpha}(R)-N\left(\varphi_{\alpha}(R)\right)^{2}
$$

The inequality implies $\varphi_{\alpha}(R) \in(0, \kappa]$. Therefore there exists a positive constant $h$ independent of $R \in(r(\alpha) / 2, r(\alpha))$ such that

$$
h \leq \sup \left\{r \geq R: \varphi_{\alpha}(\tilde{r}) \leq \kappa+1 \text { for } \tilde{r} \in[R, r]\right\}
$$

by the elliptic regularity argument. These imply that $\varphi_{\alpha}^{\prime} \geq 0$ in $\left[R_{1}, r(\alpha)\right]$ and $\lim _{r \rightarrow r(\alpha)} \varphi_{\alpha}(r)=\infty$, where $R_{1}=\max (r(\alpha) / 2, r(\alpha)-h)$, from which we get

$$
-\varphi_{\alpha}(r)+N \varphi_{\alpha}(r)^{2}>0 \quad \text { for } r \in\left[R_{2}, r(\alpha)\right)
$$

and

$$
\frac{N+1}{r}-\frac{r}{2}+r \varphi_{\alpha}(r)>0 \quad \text { for } r \in\left[R_{2}, r(\alpha)\right)
$$

with some $R_{2} \in\left[R_{1}, r(\alpha)\right)$. Applying these inequalities to (3.13), we obtain $\varphi_{\alpha}^{\prime \prime}<0$ in $\left[R_{2}, r(\alpha)\right)$. Then $\varphi_{\alpha}$ is bounded in $(0, r(\alpha))$. This contradicts $\lim \sup _{r \rightarrow r(\alpha)} \varphi_{\alpha}(r)=\infty$, which completes the proof.

Lemma 3.5 If $\alpha \in S \backslash\{\kappa\}$, then $\lim _{r \rightarrow \infty} \varphi_{\alpha}(r)=0$ and $\varphi_{\alpha}^{\prime}(r)<0$ and $\varphi_{\alpha}^{\prime \prime}(r) \geq 0$ for $r \gg 1$.

Proof. Let $r_{1}$ be the largest zero of $\varphi_{\alpha}-\kappa$ if $\varphi_{\alpha}-\kappa$ has at least one zero, and let $r_{1}=0$ if $\varphi_{\alpha}-\kappa$ has no zeros. Put

$$
G_{\alpha}(r)=r^{N+1} \exp \left(-\frac{r^{2}}{4}+\int_{r_{0}}^{r} s \varphi_{\alpha}(s) d s\right) .
$$

We see

$$
G_{\alpha}(r) \varphi_{\alpha}^{\prime}(r)=G_{\alpha}(\tilde{r}) \varphi_{\alpha}^{\prime}(\tilde{r})+\int_{\tilde{r}}^{r}\left(\varphi_{\alpha}(s)-N \varphi_{\alpha}(s)^{2}\right) G_{\alpha}(s) d s
$$

for $r, \tilde{r} \in(0, \infty)$. Suppose that $\varphi_{\alpha}^{\prime}$ has a zero $r_{2}$ in $\left(r_{1}, \infty\right)$. It follows from (3.21) that $\varphi_{\alpha}^{\prime}(r)<0$ in $\left(r_{2}, \infty\right)$ if $\varphi_{\alpha}>\kappa$ in $\left(r_{1}, \infty\right)$ and that $\varphi_{\alpha}^{\prime}(r)>0$ in $\left(r_{2}, \infty\right)$ if $\varphi_{\alpha}<\kappa$ in $\left(r_{1}, \infty\right)$. From this and Lemma 3.3, there exists a limit $C_{\alpha}=\lim _{r \rightarrow \infty} \varphi_{\alpha}(r) \in[0, \infty)$. Suppose that $C_{\alpha}=\kappa$. If $\varphi_{\alpha}^{\prime}<0$ in $\left(r_{2}, \infty\right)$, then $\varphi_{\alpha}>\kappa$ in $\left(r_{1}, \infty\right)$. Applying these 
inequalities to (3.13) yields $\varphi_{\alpha}^{\prime \prime}(r) \geq 0$ and $(N+1) / r-r / 2+r \varphi_{\alpha}(r)<0$ with some $r \in\left(r_{2}, \infty\right)$, which contradicts (3.13). If $\varphi_{\alpha}^{\prime}>0$ in $\left(r_{2}, \infty\right)$, then $\varphi_{\alpha}<\kappa$ in $\left(r_{1}, \infty\right)$. Thus we get $\varphi_{\alpha}^{\prime \prime}(r) \leq 0$ and $(N+1) / r-r / 2+r \varphi_{\alpha}(r)<0$ with some $r \in\left(r_{2}, \infty\right)$. This contradicts (3.13). Then we have $C_{\alpha} \neq \kappa$. Suppose that $C_{\alpha}>0$. The equation (3.13) can be written as

$$
\begin{aligned}
\varphi_{\alpha}(r) & -\varphi_{\alpha}(r)^{2}-\frac{2}{r} \varphi_{\alpha}^{\prime}(r)-\frac{2(N+2)}{r} \varphi_{\alpha}(r) \\
= & \varphi_{\alpha}\left(r_{2}\right)-\varphi_{\alpha}\left(r_{2}\right)^{2}-\frac{2}{r_{2}} \varphi_{\alpha}^{\prime}\left(r_{2}\right)-\frac{2(N+2)}{r_{2}} \varphi_{\alpha}\left(r_{2}\right) \\
& +\int_{r_{2}}^{r} \frac{2}{s}\left(-\varphi_{\alpha}(s)+N \varphi_{\alpha}(s)^{2}+\frac{2(N+2)}{s^{2}} \varphi_{\alpha}(s)\right) d s
\end{aligned}
$$

Take $\left\{R_{n}\right\} \subset\left[r_{2}, \infty\right)$ with $\lim _{n \rightarrow \infty} R_{n}=\infty$ such that $\lim _{n \rightarrow \infty} \varphi_{\alpha}^{\prime}\left(R_{n}\right)=0$. If $C_{\alpha} \in$ $(0, \kappa)$, then

$$
\lim _{n \rightarrow \infty}\left(\varphi_{\alpha}\left(R_{n}\right)-\varphi_{\alpha}\left(R_{n}\right)^{2}\right)=-\infty
$$

and if $C_{\alpha}>\kappa$, then

$$
\lim _{n \rightarrow \infty}\left(\varphi_{\alpha}\left(R_{n}\right)-\varphi_{\alpha}\left(R_{n}\right)^{2}\right)=\infty .
$$

This is a contradiction by Lemma 3.3. Then we get $\lim _{r \rightarrow \infty} \varphi_{\alpha}(r)=0$ and $\varphi_{\alpha}^{\prime}(r)<0$ for $r \gg 1$.

Differentiating (3.13) with respect to $r$ yields

$$
\begin{aligned}
& \varphi_{\alpha}^{\prime \prime \prime}+\left(\frac{N+1}{r}-\frac{r}{2}\right) \varphi_{\alpha}^{\prime \prime}+\left(\frac{N+1}{r}-\frac{r}{2}\right)^{\prime} \varphi_{\alpha}^{\prime}-\varphi_{\alpha}^{\prime} \\
& +\varphi_{\alpha}^{\prime}\left(r \varphi_{\alpha}^{\prime}+N \varphi_{\alpha}\right)+\varphi_{\alpha}\left(\varphi_{\alpha}^{\prime}+r \varphi_{\alpha}^{\prime \prime}+N \varphi_{\alpha}^{\prime}\right) \\
& =\varphi_{\alpha}^{\prime \prime \prime}+\left(\frac{N+1}{r}-\frac{r}{2}+r \varphi_{\alpha}\right) \varphi_{\alpha}^{\prime \prime} \\
& \quad-\left\{\frac{N+1}{r^{2}}+\frac{3}{2}-(2 N+1) \varphi_{\alpha}\right\} \varphi_{\alpha}^{\prime}+r\left(\varphi_{\alpha}^{\prime}\right)^{2} \\
& =0 .
\end{aligned}
$$

Take

$$
r_{3}=\max \left(r_{2}, \sup \left\{r \geq 0: \varphi_{\alpha}(r) \geq \frac{1}{2 N+1}\right\}\right)
$$

If there exists $r_{4} \geq r_{3}$ such that $\varphi_{\alpha}^{\prime \prime}\left(r_{4}\right)<0$, then

$$
\varphi_{\alpha}^{\prime \prime}(r) \leq \frac{1}{r^{N+1}} \exp \left(\frac{r^{2}}{4}-\frac{r^{2}}{2(2 N+1)}\right) G_{\alpha}\left(r_{4}\right) \varphi_{\alpha}^{\prime \prime}\left(r_{4}\right) \rightarrow-\infty \quad \text { as } r \rightarrow \infty .
$$


This contradicts $\lim _{r \rightarrow \infty} \varphi_{\alpha}(r)=0$. Consequently we obtain $\varphi_{\alpha}^{\prime \prime}(r) \geq 0$ for $r \gg 1$, which completes the proof.

Lemma 3.6 If $\alpha, \tilde{\alpha} \in \mathcal{S}$ and $\alpha \neq \tilde{\alpha}$, then $z\left(\varphi_{\alpha}-\varphi_{\tilde{\alpha}}\right)<\infty$ and $z\left(\varphi_{\alpha}-\varphi_{\infty}\right)<\infty$.

Proof. For $\alpha, \tilde{\alpha} \in S$ with $\alpha \neq \tilde{\alpha}$, suppose that $z\left(\varphi_{\alpha}-\varphi_{\tilde{\alpha}}\right)=\infty$ and put $\left\{r_{n}\right\}=\left\{r \in(0, \infty): \varphi_{\alpha}(r)=\varphi_{\tilde{\alpha}}(r)\right\}$. If $\left\{r_{n}\right\}$ has an accumulating point $r^{*}$, we see $\varphi_{\alpha}\left(r^{*}\right)=\varphi_{\tilde{\alpha}}\left(r^{*}\right)$ and $\varphi_{\alpha}^{\prime}\left(r^{*}\right)=\varphi_{\tilde{\alpha}}^{\prime}\left(r^{*}\right)$. This implies $\varphi_{\alpha} \equiv \varphi_{\tilde{\alpha}}$, which contradicts $\alpha \neq \tilde{\alpha}$. Then we may assume without loss of generality that $\lim _{n \rightarrow \infty} r_{n}=\infty$ and $\varphi_{\alpha}>\varphi_{\tilde{\alpha}}$ in $\left(r_{2 n}, r_{2 n+1}\right)$. Put $\Psi=\varphi_{\alpha}-\varphi_{\tilde{\alpha}}$. The function $\Psi$ satisfies

$$
\begin{aligned}
& \Psi^{\prime \prime}+\frac{N+1}{r} \Psi^{\prime}-\left(\frac{1}{2}-\kappa\right) r \Psi^{\prime}-\Psi \\
& \quad-\kappa r \Psi^{\prime}+r\left(\Psi \varphi_{\tilde{\alpha}}\right)^{\prime}+r \Psi \Psi^{\prime}+N\left(\varphi_{\alpha}+\varphi_{\tilde{\alpha}}\right) \Psi=0
\end{aligned}
$$

and

$$
\frac{1}{g}\left(g \Psi^{\prime}\right)^{\prime}-\Psi-\kappa r \Psi^{\prime}+r\left(\Psi \varphi_{\tilde{\alpha}}\right)^{\prime}+r \Psi \Psi^{\prime}+N\left(\varphi_{\alpha}+\varphi_{\tilde{\alpha}}\right) \Psi=0
$$

where $g$ is in (3.18). It follows from (3.19) that

$$
\begin{aligned}
-\left(\lambda_{j}^{\kappa}+1\right) \int_{r_{2 n}}^{r_{2 n+1}} \phi_{j}^{\kappa} g \Psi d r & =\int_{r_{2 n}}^{r_{2 n+1}}\left(\phi_{j}^{\kappa \prime} g\right)^{\prime} \Psi d r \\
& =-\int_{r_{2 n}}^{r_{2 n+1}} \phi_{j}^{\kappa \prime}\left(g \Psi^{\prime}\right) d r \\
& >\int_{r_{2 n}}^{r_{2 n+1}} \phi_{j}^{\kappa}\left(g \Psi^{\prime}\right)^{\prime} d r \quad \text { for } n \gg 1 .
\end{aligned}
$$

Take $j \geq 1$ such that $\lambda_{j}^{\kappa}>0$. Applying (3.22) to this inequality yields

$$
\begin{gathered}
\left(2+\lambda_{j}^{\kappa}\right) \int_{r_{2 n}}^{r_{2 n+1}} \Psi \phi_{j}^{\kappa} g d r+\kappa \int_{r_{2 n}}^{r_{2 n+1}} \Psi^{\prime}\left(r \phi_{j}^{\kappa} g\right) d r-\int_{r_{2 n}}^{r_{2 n+1}}\left(\Psi \varphi_{\tilde{\alpha}}\right)^{\prime}\left(r \phi_{j}^{\kappa} g\right) d r \\
-\int_{r_{2 n}}^{r_{2 n+1}} \Psi \Psi^{\prime}\left(r \phi_{j}^{\kappa} g\right) d r-N \int_{r_{2 n}}^{r_{2 n+1}}\left(\varphi_{\alpha}+\varphi_{\tilde{\alpha}}\right) \Psi\left(\phi_{j}^{\kappa} g\right) d r<0,
\end{gathered}
$$

from which we get

$$
\int_{r_{2 n}}^{r_{2 n+1}}\left\{2+\lambda_{j}^{\kappa}-N\left(\varphi_{\alpha}+\varphi_{\tilde{\alpha}}\right)\right\} \Psi \phi_{j}^{\kappa} g d r<\int_{r_{2 n}}^{r_{2 n+1}}\left(\kappa-\varphi_{\tilde{\alpha}}-\frac{1}{2} \Psi\right) \Psi\left(r \phi_{j}^{\kappa} g\right)^{\prime} d r .
$$


The right-hand side is negative and the left-hand side is positive for $n \gg 1$. This contradiction implies $z\left(\varphi_{\alpha}-\varphi_{\tilde{\alpha}}\right)<\infty$. Similarly to above, we get $z\left(\varphi_{\alpha}-\varphi_{\infty}\right)<\infty$.

Lemma 3.7 Let $N \geq 3$. For $\alpha \in \mathcal{S} \backslash\{\kappa\}, \Lambda_{\alpha} \equiv \lim _{r \rightarrow \infty} r^{2} \varphi_{\alpha}(r)$ exists in $(0, \infty)$. Moreover we have

$$
\lim _{r \rightarrow \infty} \frac{r \varphi_{\alpha}^{\prime}(r)}{\varphi_{\alpha}(r)}=-2 .
$$

Proof. For simplicity, we write $\varphi=\varphi_{\alpha}$. Putting $\rho(r)=\exp \left(-r^{2} / 4\right)$, we have

$$
-\varphi^{\prime}(r) r^{N+1} \rho(r)=\int_{r}^{\infty} \tau^{N+1} \rho\left(\varphi-\tau \varphi \varphi^{\prime}-N \varphi^{2}\right) d \tau .
$$

and

$$
-\frac{r \varphi^{\prime}(r)}{\varphi(r)}=\frac{\int_{r}^{\infty} \tau^{N+1} \rho\left(\varphi-\tau \varphi \varphi^{\prime}-N \varphi^{2}\right) d \tau}{\varphi(r) r^{N} \rho(r)} .
$$

The l'Hospital's rule yields

$$
\begin{aligned}
-\lim _{r \rightarrow \infty} \frac{r \varphi^{\prime}(r)}{\varphi(r)} & =\lim _{r \rightarrow \infty} \frac{-r^{N+1} \rho(r) \varphi(r)\left(1-r \varphi(r) \varphi^{\prime}(r)-N \varphi(r)\right)}{\left(\varphi(r) r^{N} \rho(r)\right)^{\prime}} \\
& =\lim _{r \rightarrow \infty} \frac{-1+r \varphi^{\prime}(r)+N \varphi(r)}{\left(\frac{\varphi^{\prime}(r)}{r \varphi(r)}+\frac{N}{r^{2}}-\frac{1}{2}\right)} .
\end{aligned}
$$

It follows from Lemma 3.5 and (3.13) that

$$
0>\left(-\varphi-\frac{N+1}{r^{2}}+\frac{1}{2}\right) r \varphi^{\prime}>-\varphi(r) \quad \text { for } r \gg 1 .
$$

Combining the inequality with Lemma 3.5, we have

$$
\lim _{r \rightarrow \infty} r \varphi^{\prime}(r)=0 \text { and } \lim _{r \rightarrow \infty} \frac{\varphi^{\prime}(r)}{r \varphi(r)}=0 .
$$

Hence it follows from (3.23) that

$$
-\lim _{r \rightarrow \infty} \frac{r \varphi^{\prime}(r)}{\varphi(r)}=2
$$


Therefore for any positive constant $\varepsilon$ there exists $R>0$ such that

$$
\frac{r \varphi^{\prime}(r)}{\varphi(r)}<-2+\varepsilon \text { and } \varphi(r)<R^{2-\varepsilon} r^{-2+\varepsilon} \varphi_{\alpha}(R) \quad \text { for } r \geq R .
$$

Take $k \in(0,2-\varepsilon)$. The l'Hospital's rule yields

$$
\begin{aligned}
& \lim _{r \rightarrow \infty} r^{k}\left(\frac{r \varphi^{\prime}(r)}{\varphi(r)}+2\right) \\
& =-\lim _{r \rightarrow \infty} \frac{1}{\frac{d}{d r}\left\{\varphi(r) r^{N-k} \rho(r)\right\}} \frac{d}{d r}\left\{\int_{r}^{\infty} \tau^{N+1} \rho\left(\varphi-\tau \varphi \varphi^{\prime}-N \varphi^{2}\right) d \tau-2 \varphi r^{N} \rho(r)\right\} \\
& =\lim _{r \rightarrow \infty} \frac{-r^{k+1} \varphi^{\prime}+N r^{k} \varphi+2 N r^{k-2}+2 r^{k-1}\left(\varphi^{\prime}(r) / \varphi(r)\right)}{\frac{N-k}{r^{2}}+\frac{\varphi^{\prime}(r)}{r \varphi(r)}-\frac{1}{2}}
\end{aligned}
$$

and hence

$$
\lim _{r \rightarrow \infty} r^{k}\left(\frac{r \varphi^{\prime}(r)}{\varphi(r)}+2\right)=0
$$

This implies

$$
\left|r^{k}\left(\frac{r \varphi^{\prime}(r)}{\varphi(r)}+2\right)\right|<1 \quad \text { for } r \geq R_{1}
$$

and

$$
-\frac{2}{r}-\frac{1}{r^{k+1}}<\frac{\varphi^{\prime}(r)}{\varphi(r)}<-\frac{2}{r}+\frac{1}{r^{k+1}} \quad \text { for } r \geq R_{1}
$$

with some $R_{1} \gg 1$. Therefore there exist $K_{1}, K_{2}>0$ such that

$$
\frac{K_{1}}{r^{2}}<\varphi(r)<\frac{K_{2}}{r^{2}} \quad \text { for } r \geq R_{1} \text {. }
$$

Set

$$
h(\eta)=r^{2} \varphi(r) \text { and } \eta=\log r .
$$

The equation (3.13) is written as

$$
h^{\prime \prime}+(N-4) h^{\prime}-\frac{e^{2 \eta}}{2} h^{\prime}-2(N-2) h+h h^{\prime}+(N-2) h^{2}=0 .
$$




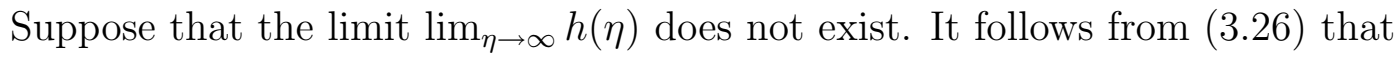

$$
K_{1} \leq \liminf _{\eta \rightarrow \infty} h(\eta)<\limsup _{\eta \rightarrow \infty} h(\eta) \leq K_{2} .
$$

Therefore there exist $\left\{\eta_{* n}\right\}$ and $\left\{\eta_{n}^{*}\right\}$ with $0<\eta_{* n}<\eta_{n}^{*}<\eta_{* n+1}$ such that $h\left(\eta_{n}^{*}\right)$ and $h\left(\eta_{* n}\right)$ are a local minimum and a local maximum, respectively. From this and (3.13), we obtain $h\left(\eta_{* n}\right)<2<h\left(\eta_{n}^{*}\right)$ for any $n$ and hence $z(h-2)=\infty$, i.e., $z\left(\varphi-\varphi_{\infty}\right)=$ $\infty$. This is a contradiction by Lemma 3.6, which implies that $\lim _{\eta \rightarrow \infty} h(\eta)$ exists. Consequently the conclusion follows from (3.26).

Lemma 3.8 Let $N \geq 3$. For $\alpha_{1}, \alpha_{2} \in \mathcal{S}$ with $\alpha_{1} \neq \alpha_{2}$, we have $\Lambda_{\alpha_{1}} \neq \Lambda_{\alpha_{2}}$.

Proof. For simplicity, we write $\Lambda_{i}=\Lambda_{\alpha_{i}}$ and $h_{i}=h_{\alpha_{i}}(i=1,2)$, where $\Lambda_{\alpha}$ is the constant in Lemma 3.7 and $h_{\alpha}$ is defined by (3.27).

Suppose that $\Lambda_{1}=\Lambda_{2}$. Put $f=h_{1} h_{2}^{\prime}-h_{1}^{\prime} h_{2}$ and

$$
g(\eta)=(N-4) \eta-\frac{1}{4} e^{2 \eta}+\int_{0}^{\eta} h_{1}(\tau) d \tau .
$$

It follows from (3.28) that

$$
\begin{gathered}
\frac{d}{d \eta}\left(e^{g(\eta)} f(\eta)\right)=e^{g(\eta)}\left[h_{1}\left\{h_{2}^{\prime \prime}+(N-4) h_{2}^{\prime}-\frac{1}{2} e^{2 \eta} h_{2}^{\prime}(\eta)+h_{1}(\eta) h_{2}^{\prime}(\eta)\right\}\right. \\
\left.-h_{2}\left\{h_{1}^{\prime \prime}+(N-4) h_{1}^{\prime}-\frac{1}{2} e^{2 \eta} h_{1}^{\prime}(\eta)+h_{1}(\eta) h_{1}^{\prime}(\eta)\right\}\right] \\
=e^{g(\eta)} h_{1}(\eta)\left(h_{1}(\eta)-h_{2}(\eta)\right)\left\{h_{2}^{\prime}(\eta)+(N-2) h_{2}(\eta)\right\} .
\end{gathered}
$$

Suppose that $\Lambda_{1}=\Lambda_{2}=2$. Since $z\left(h_{1}-2: \mathbf{R}\right)<\infty, h_{1}$ satisfies

$$
h_{1}(r)>2 \text { or } h_{1}(r)<2 \text { for } r \gg 1 \text {. }
$$

Let us consider the case where $h_{1}(r)>2$ for $r \gg 1$. For $r \gg 1, h_{1}$ does not have a local minimum and hence $h_{1}^{\prime}<0$. Combining this with (3.30) yields

$$
\frac{d}{d \eta}\left(e^{g(\eta)} \frac{d}{d \eta} h_{1}\right)<0 \quad \text { for } r \gg 1,
$$

which implies $\lim _{\eta \rightarrow \infty} h_{1}^{\prime}(\eta)=-\infty$. This contradicts Lemma 3.7. We reach a contradiction in the latter case in (3.30). Consequently we obtain $\Lambda_{1}=\Lambda_{2} \neq 2$. 
Suppose that $\Lambda_{1}=\Lambda_{2}>2$. Since $z\left(h_{1}-h_{2}: \mathbf{R}\right)<\infty$, there exists $\eta_{0} \in \mathbf{R}$ such that $h_{1}(\eta)>h_{2}(\eta)$ for $\eta \geq \eta_{0}$ or $h_{1}(\eta)<h_{2}(\eta)$ for $\eta \geq \eta_{0}$. Suppose that $h_{1}>h_{2}$ in $\left(\eta_{0}, \infty\right)$.

Since $\lim _{\eta \rightarrow \infty} h_{2}^{\prime}(\eta)=0$ by Lemma 3.7, we have

$$
\frac{d}{d \eta}\left(e^{g(\eta)} f(\eta)\right)>0 \quad \text { for } \eta \geq \eta_{1}
$$

with some $\eta_{1} \geq \eta_{0}$. Therefore there exists $\eta_{2} \geq \eta_{1}$ such that

$$
e^{g(\eta)} f(\eta)<0 \quad \text { for } \eta \geq \eta_{2}
$$

since $\lim _{\eta \rightarrow \infty} e^{g(\eta)} f(\eta)=0$. This implies that

$$
\left(\frac{h_{2}}{h_{1}}\right)^{\prime}=\frac{h_{2}^{\prime} h_{1}-h_{2} h_{1}^{\prime}}{h_{1}^{2}}=\frac{f}{h_{1}^{2}}<0 \quad \text { in }\left[\eta_{2}, \infty\right) .
$$

Thus we get

$$
\frac{\Lambda_{2}}{\Lambda_{1}}=\lim _{\eta \rightarrow \infty} \frac{h_{2}(\eta)}{h_{1}(\eta)} \leq \frac{h_{2}\left(\eta_{2}\right)}{h_{1}\left(\eta_{2}\right)}<1,
$$

which contradicts $\Lambda_{2} / \Lambda_{1}=1$.

If $h_{1}(\eta)<h_{2}(\eta)$ for $\eta \gg 1$, we define

$$
f=h_{2} h_{1}^{\prime}-h_{2}^{\prime} h_{1} \quad \text { and } \quad g=(N-4) \eta-\frac{1}{4} e^{2 \eta}+\int_{0}^{\eta} h_{2}(\tau) d \tau .
$$

Similarly to above, we obtain the same conclusion. Suppose that $\Lambda_{1}=\Lambda_{2}<2$. As mentioned in the case where $\Lambda_{1}=\Lambda_{2}>2$, we can assume that $h_{1}(\eta)<h_{2}(\eta)$ for $\eta \geq \eta_{3}$ with some $\eta_{3}$. It follows from (3.29) that

$$
\frac{d}{d \eta}\left(e^{g(\eta)} f(\eta)\right)<0 \quad \text { for } \eta \geq \eta_{3} .
$$

with some $\eta_{3}>0$. Since $\lim _{\eta \rightarrow \infty} e^{g(\eta)} f(\eta)=0$, we get

$$
e^{g(\eta)} f(\eta)>0 \quad \text { for } \eta \geq \eta_{4}
$$

with some $\eta_{4} \geq \eta_{3}$, that is, $\left(h_{2}(\eta) / h_{1}(\eta)\right)^{\prime}>0$ for $\eta \geq \eta_{4}$. Therefore we obtain

$$
\frac{\Lambda_{2}}{\Lambda_{1}}=\lim _{\eta \rightarrow \infty} \frac{h_{2}(\eta)}{h_{1}(\eta)} \geq \frac{h_{2}\left(\eta_{4}\right)}{h_{1}\left(\eta_{4}\right)}>1 .
$$

In the case where $\Lambda_{1}=\Lambda_{2}<2$ and $h_{1}(\eta)>h_{2}(\eta)$ for $\eta \gg 1$, we can treat similarly. This completes the proof.

Proposition 3.1 is now immediate from Lemmas 3.7, 3.8. 


\section{Proofs of the main theorems}

This section is devoted to the proof of Theorem 1.1. Throughout this section, let $(U, V)$ be a radial solution of (1.1) which undergoes type I blowup at $t=T$ with $B(U, V)=\{0\}$. For the corresponding solution $w$ of (1.6), let $\omega\left(w_{0}\right)$ be the omegalimit set of $w$, i.e.,

$$
\begin{aligned}
& \omega\left(w_{0}\right)=\left\{W: w\left(s_{n}\right) \rightarrow W \text { in } L_{l o c}^{\infty} \text { as } n \rightarrow \infty\right. \\
& \left.\quad \text { for a sequence }\left\{s_{n}\right\} \text { with } s_{n} \rightarrow \infty \text { as } n \rightarrow \infty\right\} .
\end{aligned}
$$

Since $|w(s)|_{\infty}$ is bounded in $\left[s_{0}, \infty\right)$, we have that $\omega\left(w_{0}\right)$ is nonempty by the parabolic regularity argument.

Lemma 4.1 Suppose that $w_{1}, w_{2} \in \omega\left(w_{0}\right)$ and $w_{1}(0)<w_{2}(0)$. If $\alpha \in I \equiv$ $\left(w_{1}(0), w_{2}(0)\right)$, then $\alpha \in \mathcal{S}$.

Proof. On the contrary, assume that there exists $\alpha \in I$ such that $\alpha \notin \mathcal{S}$. Since $r(\alpha)<\infty$, we have

$$
z\left(w(s)-\varphi_{\alpha}:[0, r(\alpha))\right) \leq k \quad \text { for any } s>s_{0}
$$

with some positive integer $k$. It is known that $z\left(w(s)-\varphi_{\alpha}:[0, r(\alpha))\right)$ is nonincreasing in $s$ and that

$$
z\left(w(s)-\varphi_{\alpha}:[0, r(\alpha))\right)<z\left(w(\widetilde{s})-\varphi_{\alpha}:[0, r(\alpha))\right) \quad \text { for } s>s_{1}>\widetilde{s}
$$

if $w\left(r_{1}, s_{1}\right)-\varphi_{\alpha}\left(r_{1}\right)=w_{r}\left(r_{1}, s_{1}\right)-\left(\varphi_{\alpha}\right)_{r}\left(r_{1}\right)=0$ at some $r_{1} \in[0, r(\alpha))$ and $s_{1}>s_{0}$. On the other hand, $w(0, s)-\varphi_{\alpha}(0)$ changes sign infinitely many times. This contradiction completes the proof.

Let $B(u)$ be the blowup set of $u$. It is immediate that $u(\xi, T) \equiv \lim _{t \rightarrow T} u(\xi, t)$ exists for $\xi \notin B(u)$.

Lemma 4.2 Suppose that $w_{1}, w_{2} \in \omega\left(w_{0}\right)$ and $w_{1}(0)<w_{2}(0)$. For $\alpha \in I \equiv$ $\left(w_{1}(0), w_{2}(0)\right)$, let

$$
u_{\alpha}(\xi, t)=(T-t)^{-1} \varphi_{\alpha}\left((T-t)^{-1 / 2} \xi\right) \quad \text { for }(\xi, t) \in[0, \infty) \times[0, T) .
$$

Then for any $\alpha \in I$ we have

$$
u(\xi, T)=u_{\alpha}(\xi, T)=\frac{\Lambda_{\alpha}}{\xi^{2}} \quad \text { for } \xi>0,
$$

where $\Lambda_{\alpha}$ is the limit in Proposition 3.1. 
Proof. On the contrary, assume that there exist $\alpha \in I$ and $\xi_{\alpha}>0$ such that $u\left(\xi_{\alpha}, T\right) \neq u_{\alpha}\left(\xi_{\alpha}, T\right)$. Then we have

$$
u\left(\xi_{\alpha}, t\right) \neq u_{\alpha}\left(\xi_{\alpha}, t\right) \quad \text { for } t \in\left[t_{\alpha}, T\right)
$$

with some $t_{\alpha} \in(0, T)$, and hence $z\left(u(t)-u_{\alpha}(t):\left[0, \xi_{\alpha}\right)\right) \leq k$ for $t \in\left[t_{\alpha}, T\right)$ with some positive integer $k$. This contradicts that $u(0, t)-u_{\alpha}(0, t)$ changes sign infinitely many times. Therefore $u(\xi, T)=u_{\alpha}(\xi, T)$ in $(0, \infty)$ for any $\alpha \in I$, which completes the proof.

The following is immediate from Proposition 3.1 and Lemma 4.2.

Lemma 4.3 If $w_{1}, w_{2} \in \omega\left(w_{0}\right)$, then $w_{1}(0)=w_{2}(0)$.

Proposition 3.1 gives an essential property for $\varphi_{\alpha}$ for $\alpha \in \mathcal{S}$, which would be also useful for other purposes. We give another proof of Lemma 4.3 without Proposition 3.1. We need the following given in [3].

Proposition 4.1 For positive constants $R, T$, let $Q_{R, T}=\left(\mathbf{R}^{N} \backslash B_{R}\right) \times[0, T]$, where $B_{R}=\left\{x \in \mathbf{R}^{N}:|x| \leq R\right\}$. Assume that u satisfies

$$
\left|\Delta u+u_{t}\right| \leq M(|u|+|\nabla u|) \quad \text { in } Q_{R, T}
$$

and

$$
|u(x, t)| \leq M \exp \left(M|x|^{2}\right) \quad \text { in } Q_{R, T}
$$

for some constant $M>0$. If $u(x, 0)=0$ for any $x \in \mathbf{R}^{N} \backslash B_{R}$, then $u$ vanishes identically in $Q_{R, T}$.

Another proof of Lemma 4.3 The conclusion is trivial if the solution $u$ under consideration is a backward self-similar solution. Therefore we may suppose without loss of generality that $u$ is not a backward self-similar solution. Assume that there exist $w_{1}, w_{2} \in \omega\left(w_{0}\right)$ such that $w_{1}(0) \neq w_{2}(0)$. Then we get a contradiction by Lemma 4.2 and Proposition 4.1,

According to Lemma 4.3, there exists $K \in \mathbf{R}$ such that

$$
w(0, s) \rightarrow K \quad \text { as } s \rightarrow \infty .
$$


Lemma 4.4 For each positive integer $k$, we have

$$
\frac{\partial^{k} w}{\partial s^{k}}(0, s) \rightarrow 0 \quad \text { as } s \rightarrow \infty .
$$

Proof. On the contrary, we assume that there exist $k,\left\{s_{n}\right\} \subset\left[s_{0}, \infty\right)$ and $\delta>0$ such that $\lim _{n \rightarrow \infty} s_{n}=\infty$ and $\left|\partial^{k} w / \partial s^{k}\left(0, s_{n}\right)\right|>\delta$ for $n$. We assume without loss of generality that $\lim _{s \rightarrow \infty} \partial^{i} w / \partial s^{i}(0, s)=0$ for $i=1,2,3, \cdots, k-1$ if $k \geq 2$ and that $\partial^{k} w / \partial s^{k}\left(0, s_{n}\right)>\delta$ for $n$. By the parabolic regularity argument, there exist $C_{1}>0$ and $S_{1}>s_{0}$ such that

$$
\left|\frac{\partial^{k+1} w}{\partial s^{k+1}}(0, s)\right| \leq C_{1} \quad \text { for } s \geq S_{1} .
$$

Let $K_{k}=K$ with the constant $K$ in (4.32) if $k=1$ and let $K_{k}=0$ if $k \geq 2$. For $\varepsilon \in\left(0, \delta^{2} /\left(8 C_{1}\right)\right)$ there exists $S_{2} \geq S_{1}$ such that

$$
\left|\frac{\partial^{k-1} w}{\partial s^{k-1}}(0, s)-K_{k}\right|<\varepsilon \quad \text { for } s \geq S_{2} .
$$

Putting $\tilde{s}_{n}=s_{n}+\delta / C_{1}$, we see

$$
\begin{aligned}
\frac{\partial^{k-1} w}{\partial s^{k-1}}\left(0, \tilde{s}_{n}\right) & =\frac{\partial^{k-1} w}{\partial s^{k-1}}\left(0, s_{n}\right)+\frac{\partial^{k} w}{\partial s^{k}}\left(0, s_{n}\right)\left(\tilde{s}_{n}-s_{n}\right)+\frac{1}{2} \frac{\partial^{k+1} w}{\partial s^{k+1}}\left(0, \tau_{n}\right)\left(\tilde{s}_{n}-s_{n}\right)^{2} \\
& \geq K_{k}-\varepsilon+\delta\left(\tilde{s}_{n}-s_{n}\right)-\frac{C_{1}}{2}\left(\tilde{s}_{n}-s_{n}\right)^{2} \\
& \geq K_{k}+\frac{3 \delta^{2}}{8 C_{1}} \\
& >K_{k}+3 \varepsilon
\end{aligned}
$$

for some $\tau_{n} \in\left(s_{n}, \tilde{s}_{n}\right)$. This contradicts (4.33), which completes the proof.

Lemma 4.5 For any nonnegative integers $i, j$, we have

$$
\frac{\partial^{i+j+1}}{\partial r^{j} \partial s^{i+1}} w(0, s) \rightarrow 0 \quad \text { as } s \rightarrow \infty .
$$

Proof. For $X \in \mathbf{R}^{N+2}$ with $|X|=r$, put $\tilde{w}(X, s)=w(r, s)$. The function $\tilde{w}$ is a solution to

$$
\tilde{w}_{s}=\Delta_{X} \tilde{w}-\frac{X}{2} \cdot \nabla_{X} \tilde{w}-\tilde{w}+\tilde{f}(X, \tilde{w}) \quad \text { in } \mathbf{R}^{N+2} \times\left(s_{0}, \infty\right),
$$


where

$$
\Delta_{X}=\sum_{i=1}^{N+2} \frac{\partial^{2}}{\partial X_{i}^{2}}, \quad \nabla_{X}=\left(\frac{\partial}{\partial X_{1}}, \frac{\partial}{\partial X_{2}}, \cdots, \frac{\partial}{\partial X_{N+2}}\right)
$$

and

$$
\tilde{f}(X, \tilde{w})=\tilde{w}\left(X \cdot \nabla_{X} \tilde{w}+N \tilde{w}\right)
$$

for $X=\left(X_{1}, X_{2}, \cdots, X_{N+2}\right) \in \mathbf{R}^{N+2}$. Since $\tilde{w}$ is radial and smooth, $w$ satisfies

$$
\frac{\partial^{j}}{\partial r^{j}}\left(\frac{\partial^{i}}{\partial s^{i}} w(0, s)\right)=0 \quad \text { for } s \in\left(s_{0}, \infty\right), j=1,3,5, \cdots \quad \text { and } i=0,1,2, \cdots
$$

Differentiating (4.34) with respect to $s$ yields

$$
\frac{\partial^{i+1}}{\partial s^{i+1}} \tilde{w}=\Delta_{X} \frac{\partial^{i}}{\partial s^{i}} \tilde{w}-\frac{X}{2} \cdot \nabla_{X} \frac{\partial^{i}}{\partial s^{i}} \tilde{w}-\frac{\partial^{i}}{\partial s^{i}} \tilde{w}+\frac{\partial^{i}}{\partial s^{i}} \tilde{f}(X, \tilde{w}) \quad \text { for } i \geq 1 .
$$

According to Lemma 4.4, it follows from (4.35) and (4.36) that

$$
\lim _{s \rightarrow \infty} \Delta_{X} \frac{\partial^{i}}{\partial s^{i}} \tilde{w}(0, s)=0 \quad \text { for } i \geq 1
$$

Let $J$ be a positive integer. We assume that

$$
\lim _{s \rightarrow \infty} \Delta_{X}^{j} \frac{\partial^{i}}{\partial s^{i}} \tilde{w}(0, s)=0 \quad \text { for } j=0,1,2,3, \cdots, J \text { and } i \geq 1 .
$$

Operating $\Delta_{X}^{J}$ for (4.36) yields

$$
\begin{array}{r}
\Delta_{X}^{J} \frac{\partial^{i+1}}{\partial s^{i+1}} \tilde{w}=\Delta_{X}^{J+1} \frac{\partial^{i}}{\partial s^{i}} \tilde{w}-\Delta_{X}^{J}\left(\frac{X}{2} \cdot \nabla_{X} \frac{\partial^{i}}{\partial s^{i}} \tilde{w}\right) \\
-\Delta_{X}^{J}\left(\frac{\partial^{i}}{\partial s^{i}} \tilde{w}\right)+\Delta_{X}^{J}\left(\frac{\partial^{i}}{\partial s^{i}} \tilde{f}(X, \tilde{w})\right) .
\end{array}
$$

For $j=0,1,2,3, \cdots, J$ and $i \geq 1$, it follows from (4.37) that

$$
\begin{aligned}
\lim _{s \rightarrow \infty} \Delta_{X}^{j}\left(\frac{X}{2} \cdot \nabla_{X} \frac{\partial^{i}}{\partial s^{i}} \tilde{w}(0, s)\right) & =\lim _{s \rightarrow \infty} \frac{1}{2}(N+2)^{j}{ }_{2 j} C_{1} \frac{\partial^{2 j}}{\partial r^{2 j}}\left(\frac{\partial^{i}}{\partial s^{i}} w(0, s)\right) \\
& =\lim _{s \rightarrow \infty} j \Delta_{X}^{j} \frac{\partial^{i} \tilde{w}}{\partial s^{i}}(0, s) \\
& =0 .
\end{aligned}
$$


Combining this with (4.36) and (4.35) yields

$$
\begin{aligned}
& \lim _{s \rightarrow \infty} \Delta_{X}^{J}\left(\frac{\partial^{i}}{\partial s^{i}} \tilde{f}(0, \tilde{w}(0, s))\right) \\
& \quad=\lim _{s \rightarrow \infty} \Delta_{X}^{J}\left(\frac{X}{2} \cdot \nabla_{X} \frac{\partial^{i} \tilde{w}^{2}}{\partial s^{i}}(0, s)\right)+\lim _{s \rightarrow \infty} \frac{1}{2} \Delta_{X}^{J}\left(\frac{\partial^{i} \tilde{w}^{2}}{\partial s^{i}}(0, s)\right) \\
& \quad=0
\end{aligned}
$$

for $i \geq 1$. Those imply $\lim _{s \rightarrow \infty} \Delta_{X}^{J+1} \frac{\partial^{i} \tilde{w}}{\partial s^{i}}(0, s)=0$ for $i \geq 1$, which completes the proof.

We are now in a position to prove Theorem 1.1.

\section{Proof of Theorem 1.1 Let}

$$
\begin{aligned}
\mathcal{V}=\left\{V: w_{s}\left(s_{n}\right)\right. & \rightarrow V \text { in } L_{l o c}^{\infty} \text { as } n \rightarrow \infty \\
& \text { for a sequence } \left.\left\{s_{n}\right\} \text { with } s_{n} \rightarrow \infty \text { as } n \rightarrow \infty\right\} .
\end{aligned}
$$

Take $V \in \mathcal{V}$ arbitrarily. By the parabolic regularity argument, for each nonnegative integer $i$ there exists a sequence $\left\{s_{n}\right\}$ with $s_{n} \rightarrow \infty$ as $n \rightarrow \infty$ such that

$$
w_{s}\left(s_{n}\right) \rightarrow V \quad \text { in } C_{l o c}^{j} \text { as } n \rightarrow \infty .
$$

Therefore we have

$$
\frac{d^{j}}{d r^{j}} V(0)=0 \quad \text { for } j=1,2,3, \cdots
$$

from Lemma 4.5. By the Taylor expansion, we get

$$
\begin{aligned}
w_{s}(r, s)= & w_{s}(0, s)+\frac{\partial}{\partial r} w_{s}(0, s) \cdot r+\frac{1}{2} \frac{\partial^{2}}{\partial r^{2}} w_{s}(0, s) \cdot r^{2} \\
& +\cdots+\frac{1}{(k-1) !} \frac{\partial^{k-1}}{\partial r^{k-1}} w_{s}(0, s) \cdot r^{k-1}+\frac{1}{k !} \frac{\partial^{k}}{\partial r^{k}} w_{s}(r \theta, s) \cdot r^{k}
\end{aligned}
$$

with some $\theta \in(0,1)$ for $r>0$ and $s>s_{0}$. According to Proposition 2.1, there exist $S>s_{0}$ and $R>0$ such that

$$
\sup \left\{\frac{1}{k !}\left|\frac{\partial^{k}}{\partial r^{k}} w_{s}(r, s)\right| \cdot r^{k}: r \in(0, R) \text { and } s \geq S\right\} \rightarrow 0 \quad \text { as } k \rightarrow \infty .
$$


Therefore it follows from (4.39) and (4.41) that

$$
\begin{aligned}
V(r)= & V(0)+\frac{d}{d r} V(0) \cdot r+\frac{1}{2} \frac{d^{2}}{d r^{2}} V(0) \cdot r^{2} \\
& +\cdots+\frac{1}{(k-1) !} \frac{d^{k-1}}{d r^{k-1}} V(0) \cdot r^{k-1}+\frac{1}{k !} \frac{d^{k}}{d r^{k}} V(r \theta) \cdot r^{k}
\end{aligned}
$$

for $r>0$. Since

$$
\frac{1}{k !} \frac{d^{k}}{d r^{k}} V(r \theta) \cdot r^{k} \rightarrow 0 \quad \text { as } k \rightarrow \infty
$$

by (4.42), we see that $V$ is analytic at $r=0$. Thus it follows from (4.40) and (4.43) that $V(r)=0$ for $r \geq 0$. Since for each $W \in \omega\left(w_{0}\right)$ there exists a sequence $\left\{s_{n}\right\}$ with $s_{n} \rightarrow \infty$ as $n \rightarrow \infty$ such that $w\left(s_{n}\right) \rightarrow W$ and $w_{s}\left(s_{n}\right) \rightarrow 0$ in $C_{l o c}^{2}$ as $n \rightarrow \infty$, we get

$$
W_{r r}+\frac{N+1}{r} W_{r}-\frac{r}{2} W_{r}-W+W\left(r W_{r}+N W\right)=0 \quad \text { in }(0, \infty) .
$$

Since $W(0)$ is constant for $W \in \omega\left(w_{0}\right)$ by Lemma 4.3 , we obtain $\omega\left(w_{0}\right)=\left\{\varphi_{\alpha}\right\}$ for some $\alpha \in \overline{\mathcal{S}}$. This means that $w(s) \rightarrow \varphi_{\alpha}$ in $L_{l o c}^{\infty}$ as $s \rightarrow \infty$.

Let $u$ be defined in (1.4). Under an additional assumption (1.8), we see

$$
u(0, t)=|u(t)|_{\infty} \geq \frac{1}{N}(T-t)^{-1} \quad \text { in }[0, T)
$$

by the comparison theorem, and hence $w(0, s) \geq 1 / N$ for $s \geq s_{0}$. This implies that $\alpha \in \mathcal{S}$.

Acknowledgement Y. Giga is partly supported by the Grant-in-Aid for Scientific Research (S) No.21224001, JSPS and the Grant-in-Aid for challenging Exploratory Research No.20654017, JSPS. N. Mizoguchi is supported by JST PRESTO program. T. Senba is supported by the Grant-in-Aid for Scientific Research (C) No.18540189, JSPS.

\section{References}

[1] W. W. Bell, Special functions for scientists and engineers (1968), London, Van Nostrand.

[2] P. Biler, D. Hilhorst and T. Nadzieja, Existence and nonexistence of solutions for a model gravitational of particles. Colloq. Math. 67 (1994), 297-308. 
[3] L. Escauriaza, G. Seregin and V. Šverák, Backward uniqueness for parabolic equations, Arch. Rational Mech. Anal. 169 (2003), 147-157.

[4] A. Friedman, On the regularity of the solutions of nonlinear elliptic and parabolic systems of partial differential equations, J. Math. Mech. 7 (1958), 43-59.

[5] A.Friedman, Partial differential equations of parabolic type, Prentice Hall, 1964.

[6] I. A. Guerra and M. A. Peletier, Self-similar blow-up for a diffusion-attraction problem, Nonlinearity 17 (2004), 2137-2162.

[7] M.A.Herrero, E. Medina and J.J.L. Velázquez, Finite-time aggregation into a single point in a reaction-diffusion system, Nonlinearity 10 (1997), 1739-1754.

[8] _ Self-similar blowup for a reaction-diffusion system, Journal of Computational and Applied Mathematics 97 (1998), 99-119.

[9] M.A. Herrero and J.J.L. Velázquez, A blow-up mechanism for a chemotaxis model, Ann. Scoula Norm. Sup. Pisa IV 35 (1997), 633-683.

[10] _ A blow up result for semilinear heat equations in the supercritical case, preprint.

[11] S. Itô, Diffusion equations, Anew. Math. Soc, (1992), (Japanese original(1979)).

[12] E.F. Keller and L.A. Segel, Initiation of slime mold aggregation viewed as an instability, J. Theor. Biol. 26 (1970), 399-415.

[13] O. A.Ladyzhenskaya, V. A. Solonnikov and N. N. Uralt'seva, Linear and Quasilinear Equations of Parabolic Type (1967), Moscow, Nauka (Russian original) : English translation; Amer. Math. Soc. (1968), Providence, R.I.

[14] N. Mizoguchi, Type II blowup for a semilinear heat equation. Adv. Diff. Eqns. 9 (2004), 1317-1338.

[15] Y. Naito and T. Senba, Blow-up behavior of solutions to a chemotaxis system on higher dimensional domains, preprint.

[16] T. Senba, Blowup behavior of radial solutions to Jäger-Luckhaus system in high dimensional domains, Funkcial. Ekvac. 48 (2005), 247-271.

[17] T. Senba, A fast blowup solution to an elliptic-parabolic system related to chemotaxis, Adv. Diff. Eqns. 11 (2006), 981-1030. 
[18] T. Suzuki, Semilinear Elliptic Equations (1994), Tokyo, Gakkoutosho.

[19] T. I. Zelenyak, Stabilization of solutions of boundary value problems for a second order parabolic equation with one space variable, Diff. Eqns. 4 (1968), 17-22.

\section{Yoshikazu Giga}

Graduate School of Mathematical Sciences, University of Tokyo,

Komaba 3-8-1, Meguro-ku, Tokyo 153-8914, Japan

(labgiga@ms.u-tokyo.ac.jp),

Noriko Mizoguchi

Department of Mathematics, Tokyo Gakugei University, Koganei, Tokyo 184-8501, Japan

(mizoguti@u-gakugei.ac.jp)

and

Precursory Research for Embryonic Science and Technology (PRESTO),

Japan Science and Technology Agency (JST),

4-1-8 Honcho Kawaguchi, Saitama 332-0012, Japan,

TAKASI SENBA

Faculty of Engineering, Kyushu Institute of Technology, 1-1 Sensui-cho Tobata-ku Kitakyushu-shi, 804-8550, Japan

(senba@mns.kyutech.ac.jp) 